Service social

\title{
Une intervention préventive pour les couples qui vivent la recomposition de leur famille
}

\section{Claudine Parent et Madeleine Beaudry}

Volume 46, numéro 2-3, 1997

Groupes - Symposium 1997

URI : https://id.erudit.org/iderudit/706769ar

DOI : https://doi.org/10.7202/706769ar

Aller au sommaire du numéro

Éditeur(s)

École de service social de l'Université Laval

ISSN

1708-1734 (numérique)

Découvrir la revue

Citer cet article

Parent, C. \& Beaudry, M. (1997). Une intervention préventive pour les couples qui vivent la recomposition de leur famille. Service social, 46(2-3), 241-260. https://doi.org/10.7202/706769ar
Résumé de l'article

Cet article présente les fondements théoriques et les principales composantes d'un programme d'intervention préventive conçu pour les couples qui vivent la recomposition de leur famille. Les auteures expliquent comment elles allient la méthode individuelle à la méthode de groupe dans cette intervention et précisent l'utilité de cette double approche pour les couples de familles recomposées. Ce programme fait l'objet d'une préexpérimentation (automne 1997) et sera évalué à l'hiver 1998. À cet effet, les auteures décrivent brièvement la méthode d'évaluation utilisée. 


\title{
Une intervention préventive pour les couples qui vivent la recomposition de leur famille 1
}

\author{
Claudine PARENT \\ Madeleine BEAUDRY
}

Cet article présente les fondements théoriques et les principales composantes d'un programme d'intervention préventive conçu pour les couples qui vivent la recomposition de leur famille. Les auteures expliquent comment elles allient la méthode individuelle à la méthode de groupe dans cette intervention et précisent l'utilité de cette double approche pour les couples de familles recomposées. Ce programme fait l'objet d'une préexpérimentation (automne 1997) et sera évalué à l'hiver 1998. À cet effet, les auteures décrivent brièvement la méthode d'évaluation utilisée.

This article discusses the theoretical background and main components of a preventive intervention program designed for couples living in a stepfamily. The authors explain how they combine the individual method to the group method in this intervention and state the usefulness of this double approach for the couples from stepfamilies. This program is undergoing a preexperimental phase (autumn 1997) and will be assessed during the winter months of 1998. For that purpose, the authors briefly describe the assessment method that will be used.

1. Cette recherche est rendue possible grâce à des bourses de la Fondation de I'Université Laval (1995-1996), de la Fondation AFDU-Québec de la Faculté des sciences sociales de l'Université Laval (1995-1996), du Fonds de soutien de revenu des étudiants au doctorat en service social de l'Université Laval (1995-1997) et du Conseil québécois de la recherche sociale (CQRS-19961999) qui ont été accordées à la première auteure. Une subvention du fonds pour la Formation de chercheurs et l'aide à la recherche (FCAR) accordée à la deuxième auteure a également contribué à la mise sur pied de ce projet. 


\section{INTRODUCTION}

Le paysage familial nord-américain est marqué par le divorce et la séparation de près de $50 \%$ des couples mariés ${ }^{2}$. Ce phénomène a donné lieu à une augmentation notable de la famille dite recomposée, soit une famille dans laquelle au moins un adulte $a$ un ou des enfants issus d'une précédente union (Ganong et Coleman, 1994). Au Canada, on estime que, depuis la fin des années 1980, $20 \%$ des mariages enregistrés sont en fait des remariages (Statistique Canada, 1994). Au Québec, le nombre de familles recomposées est passé de $5,4 \%$ en 1987 à $8,4 \%$ en 1992-1993 (Bellerose, Lavallée et Camirand, 1994).

Par ailleurs, ces recompositions familiales apparaissent encore plus fragiles que les premières unions. Des résultats de recherches indiquent que le taux de séparation des secondes unions est plus élevé, qu'il est vraisemblablement associé à la présence des enfants nés de l'union précédente et que la séparation survient plus rapidement (Lawton et Sanders, 1994; Wineberg, 1992; White et Booth, 1985). À ce sujet, une étude américaine révèle que $17 \%$ des couples qui ont des enfants nés d'une précédente union sont divorcés trois ans après le remariage, contre $9 \%$ des couples n'ayant pas eu d'enfants de l'union antérieure (Cherlin, 1987). Au Canada, Desrosiers, Le Bourdais et Laplante (1995) soutiennent que près d'un couple sur cinq mettrait fin à sa relation cinq ans après avoir formé une famille recomposée. En parallèle, plusieurs cliniciens affirment que les difficultés vécues par les couples de familles recomposées comportent un potentiel de risque important pour la stabilité de la famille (Ganong et Coleman, 1994; Fortier et Marino, 1990; Visher et Visher, 1990). Compte tenu de la probabilité encore plus élevée d'une rupture conjugale après une recomposition familiale et, par conséquent, du risque que les parents et leurs enfants soient exposés à nouveau aux effets néfastes d'une telle situation, les conjoints doivent pouvoir bénéficier de services avant que les problèmes deviennent irréversibles. Le Conseil de la famille (1995) souligne notamment la nécessité « d'un soutien et d'une solidarité envers ceux qui veulent recomposer une famille dans les meilleures conditions

2. Le Secrétariat à la famille (1995) indique qu'en 1990 au Québec la proportion des mariages qui se terminaient par un divorce était de $49,4 \%$. 
personnelles et sociales possible parce que c'est toute la société qui pourrait en profiter » (p. 4). Le projet de mettre en place un programme d'intervention préventive adapté aux besoins des couples qui vivent la recomposition de leur famille s'inscrit dans cette foulée. II a pris naissance après plusieurs années de recherche et d'intervention auprès des couples et, plus récemment, des familles recomposées. Cet article présente les fondements théoriques qui ont guidé les auteures dans l'élaboration de ce programme. Il décrit également les principales composantes de l'intervention et il explique l'utilité d'une méthode mixte (individuelle/groupe) dans le domaine de la recomposition familiale. Finalement, les auteures donnent un bref aperçu de la méthode d'évaluation qui sera utilisée à l'hiver 1998 et qui servira à vérifier les effets de ce programme.

\section{LES FONDEMENTS THÉORIQUES DU PROGRAMME}

Ce programme préventif, d'orientation behaviorale cognitive, vise autant à prévenir la détresse conjugale qu'à favoriser l'adaptation des couples-parents et de leurs enfants à la recomposition familiale. Sa conception repose sur des connaissances issues des études et des recherches qui portent sur les familles recomposées et sur celles qui s'intéressent à la prévention de la détresse dans un couple. Mentionnons brièvement les principaux éléments qui ont guidé l'élaboration du programme.

L'évolution constante du modèle behavioral cognitif est fondée sur la recherche empirique. Dans ce modèle, l'étude des relations conjugales s'inspire de la théorie de l'apprentissage social. Cette théorie est constituée d'un ensemble de modèles théoriques, tels que le modèle du conditionnement opérant, la théorie de l'échange social, la théorie de la communication et la théorie cognitive. L'application de ces différents modèles a guidé un grand nombre de recherches sur les échanges familiaux et conjugaux. Les résultats de ces recherches permettent de faire ressortir quatre hypothèses principales pour expliquer les difficultés conjugales:

1. Les difficultés dans les relations de couple sont le fruit d'un faible degré de renforcement positif.

2. L'utilisation du contrôle aversif (blâmes, menaces, ordres) pour modifier les comportements du partenaire provoque le retrait de celui-ci et la diminution du renforcement positif. 
3. Les conjoints satisfaits communiquent plus adéquatement que les couples insatisfaits. Ils possèdent les habiletés nécessaires pour transmettre des messages clairs et saisir fidèlement les messages du conjoint. Les conjoints insatisfaits sont plus négatifs et moins constructifs dans leurs échanges. Ils présentent un moins grand répertoire de réponses aux remarques négatives et saisissent moins bien les messages non verbaux.

4. Sur le plan cognitif, "Les couples en difficulté [...] ont une tendance marquée à discréditer le comportement positif de leur conjoint en en attribuant la cause à des circonstances extérieures ou à l'état de leur conjoint. [...] Même quand ce comportement a un effet bénéfique, le conjoint insatisfait croit que son partenaire a agi de manière non intentionnelle ou à cause de circonstances particulières et qu'il est peu probable qu'il agisse de la même façon à l'avenir. Par contre, quand il s'agit d'un comportement négatif, le conjoint insatisfait en attribue la cause aux traits de personnalité de son partenaire et croit qu'il a agi volontairement. [...] En somme, il semble porter une attention sélective aux événements négatifs, et attribuer les difficultés à l'attitude négative du partenaire à son égard " (Beaudry et Boisvert, 1988, p. 47-49).

En résumé, cinq principaux facteurs expliquent les difficultés conjugales : a) individuels (incompatibilités sur le plan des goûts et des valeurs); b) environnementaux (changements dus, par exemple, à la naissance d'un enfant ou à la perte d'un emploi); c) cognitifs (attention sélective, attribution d'intentions, croyances et attentes de comportements); d) comportementaux (habiletés de communication et de résolution de problèmes) et e) relationnels (renforcements mutuels des comportements). Les habiletés de communication sont reconnues comme étant le facteur qui a le plus de poids dans la distinction entre les couples satisfaits et les couples insatisfaits. Voilà pourquoi les programmes de prévention doivent accorder une importance primordiale au développement des habiletés de communication.

Voyons maintenant comment ces différents facteurs peuvent s'appliquer à un contexte de recomposition familiale. Les premières années de vie dans une famille recomposée comportent plusieurs sources de tensions et de stress (Fausse, 1995; Crosbie-Burnett, 1989). Les adultes aussi bien que les enfants arrivent soudainement 
ensemble avec une foule d'expériences et d'émotions différentes issues de leurs expériences passées. De nouveaux rôles, règles et traditions ont besoin d'être créés, négociés et implantés, ce qui rend les frictions quasi inévitables (Visher et Visher, 1990). De plus, la présence d'enfants nés d'unions précédentes implique souvent la réorganisation des horaires de visite, des périodes de garde et du partage des responsabilités; elle suppose qu'on entretient des relations avec un parent biologique extérieur à la famille et avec une exbelle-famille (les grands-parents biologiques des enfants, les oncles, les tantes, etc.); elle oblige à définir le rôle du beau-parent (parent non biologique des enfants) à l'égard de ces enfants et finalement, dans une famille recomposée complexe ${ }^{3}$, elle demande l'harmonisation des rapports entre des enfants issus de mariages différents. Le fait de vivre dans une famille recomposée modifie également les rapports entre les parents biologiques et leurs enfants. On observe fréquemment chez ces derniers des problèmes de loyauté vis-à-vis de l'un ou l'autre des parents ainsi que la crainte de perdre l'affection et l'attention de l'un des parents (Duncan et Brown, 1992; Whitsett et Land, 1992). Enfin, les conjoints de familles recomposées doivent trouver des solutions individuelles à leurs difficultés, puisque les modèles traditionnels ne constituent pas toujours des réponses idéales pour ce contexte familial particulier ${ }^{4}$ (Lawton et Sanders, 1994; Parent, 1994; Hetherington, Cox et Cox, 1985). Plusieurs auteurs soutiennent que ces situations exigent, de la part des conjoints, de très bonnes habiletés de communication (Lawton et Sanders,

3. La famille recomposée complexe est une famille dans laquelle les deux adultes ont au moins un enfant né d'une union antérieure. Par opposition, la famille recomposée simple est celle dans laquelle un seul des deux adultes a un ou des enfants nés d'une précédente union (Clingempeel et collab., 1984).

4. Par exemple, dans les modèles traditionnels d'intervention conçus pour les couples, les deux conjoints sont encouragés à prendre une part active dans la discipline et l'éducation des enfants. II n'est pas évident que ce soit la solution idéale pour les couples-parents en famille recomposée. En effet, le beau-parent n'est peut-être pas disposé à jouer un rôle aussi actif auprès des enfants de son partenaire ou de sa partenaire (Lawton et Sanders, 1994). De plus, certaines études suggèrent que le beau-parent qui tient un rôle de collaborateur dans l'éducation des enfants a de meilleures relations avec eux que celui qui s'attribue le rôle de premier responsable de la discipline dans la famille (Parent, 1994; Hetherington et collab., 1985). 
1994; Parent, 1994; O'Flaherty et Workman Eells, 1993; Pasley, Koch et Ihinger-Tallman, 1993; Skopin, Newman et McKenry, 1993; Visher et Visher, 1990). Par exemple, Saint-Jacques (1993) note que la clarté dans l'expression des attentes et la présence d'un consensus entre les conjoints quant à celles-ci sont deux facteurs importants associés à la tension de rôle dans les familles recomposées. D'autres études portant sur les prises de décision et les accords qui sont négociés par le couple-parent au sujet des enfants démontrent qu'ils influencent significativement l'établissement de la relation entre le beau-parent et l'enfant (Skopin et collab., 1993; Palisi, Orleans, Caddell et Korn, 1991; Hobart, 1988). Finalement, à la suite d'une revue des écrits visant à identifier les facteurs contribuant aux problèmes de comportement chez les enfants dans le contexte d'une recomposition familiale, Lawton et Sanders (1994) concluent que les problèmes à l'intérieur de ces familles sont causés et maintenus par des processus d'interaction familiale inadéquats et les déficits d'habiletés préexistants.

Le programme préventif que nous proposons est basé sur l'enseignement systématique d'habiletés de communication et de résolution de problèmes. II suggère d'assister les couples-parents dans la négociation des difficultés inhérentes à la recomposition familiale et de les assister dans le développement d'habiletés et de stratégies pour améliorer les relations et créer un environnement familial solide. Plusieurs recherches dans le domaine de la prévention ont démontré que l'expression des attentes et l'enseignement d'habiletés de communication et de résolution de problèmes sont des composantes importantes pour la prévention de la détresse conjugale et familiale. Par exemple, des styles d'interaction non fonctionnels, tels le retrait et les contrôles aversifs, peuvent compromettre le développement fonctionnel et émotif de certains enfants (Markman, 1991). Pour ce qui est des conjoints, les difficultés de communication peuvent engendrer plusieurs conséquences : sentiments de colère, d'anxiété ou de dépression, perceptions erronées, attribution d'intentions malicieuses, irrespectueuses ou négligentes à l'endroit des autres membres de la famille (Leslie et Epstein, 1988). Par ailleurs, lors de la recomposition d'une famille, les couples font face à de nouvelles demandes et doivent souvent assumer de nouveaux rôles. Dans cette situation, il arrive fréquemment que les conjoints se sentent dépassés et ne sachent pas comment agir. Pour Floyd, 
Markman, Kelly, Blumberg et Stanley (1995), « les habiletés de communication, les croyances réalistes à propos de la relation et la confiance de résoudre les désaccords futurs sont des ressources qui peuvent amoindrir la tâche de négocier avec le stress des nouvelles situations [entendons ici la recomposition familiale] " (p. 215). En outre, les auteurs soulignent que la qualité de l'ajustement établi lors d'une transition influence l'éventail des nouvelles possibilités ou des stresseurs rencontrés plus tard. En conséquence, plus une intervention est offerte tôt, plus les couples ont accès à des possibilités qui peuvent leur éviter les stresseurs significatifs présents et à venir.

II reste, cependant, que les exigences nécessaires à la résolution de certaines difficultés vécues par les couples de familles recomposées dépassent l'apprentissage de techniques de résolution de problèmes. Ainsi, les couples doivent accepter de vivre une vie de couple différente de celle qui avait été imaginée, accepter que les liens entre enfants et beaux-parents soient différents de ceux qui existent entre les enfants et leurs parents naturels, accepter les styles de vie et les valeurs différentes des autres membres de la famille, etc. II n'est pas rare que ces difficultés se traduisent par des blâmes sur ce que l'autre conjoint ou conjointe devrait faire ou ne pas faire pour atténuer ces situations. Pour favoriser l'acceptation de ces situations et sortir les conjoints de leur lutte pour changer l'autre, le programme offre de l'information pertinente sur la vie conjugale et familiale dans un contexte de recomposition. Ces informations visent, entre autres choses, à ce que les conjoints puissent se construire une perspective de vie familiale différente de celle d'une famille nucléaire. Dans ce domaine, les difficultés proviennent souvent de l'écart entre les référents culturels de la famille en tant que famille nucléaire et la réalité familiale de la famille recomposée. Cela laisse sousentendre que tout ce qui dévie du modèle de la famille nucléaire n'est pas une " vraie " famille ou, du moins, n'est pas aussi valable. En effet, l'image traditionnelle de la famille où tous les membres occupent une seule maison (un couple de parents et leurs enfants) s'oppose diamétralement à celle d'une famille où un parent habite à l'extérieur de la famille alors qu'un adulte non parent de même sexe vit dans la maison. De plus, il existe peu de normes socialement acceptées pour savoir qui fait partie de la famille et qui n'en fait pas partie, comme il n'y a pas de terme spécifique dans la langue française pour nommer cette famille ou les personnes qui la composent. 
Par exemple, mes parents, mes frères et mes sœurs deviennent-ils respectivement les grands-parents, les oncles et les tantes des enfants de mon partenaire? Doit-on dire famille recomposée, reconstituée ou restructurée? Les termes de " belle-mère » ou de « beaupère " réfèrent-ils à la belle-mère ou au beau-père des enfants ou aux parents du conjoint (Jacobson, 1990)?

La façon traditionnelle de concevoir la famille n'aide pas les conjoints à accepter les différences car, dans cette perspective, elles ne peuvent faire partie de la vie familiale dite normale. Les informations portant sur la vie en famille recomposée et sur ce qu'il est naturel d'y rencontrer comme difficultés permettent de rassurer les conjoints quant à ce qu'ils peuvent vivre ou ressentir dans leur famille. De plus, les informations offertes dans le programme mettent aussi l'accent sur les forces d'une famille recomposée; ce qui favorise la construction d'une perspective de la famille qui est différente, mais tout aussi valable que celle de la famille traditionnelle. Dans un contexte familial encore relativement nouveau, ces connaissances constituent un atout précieux.

\section{LE PROGRAMME D'INTERVENTION}

Le présent projet s'inspire d'un programme d'intervention préventive, The Prevention and Relationship Enhancement Program (PREP), élaboré au départ pour les jeunes couples qui projetaient de se marier (Markman, 1981). Les fondements de ce programme s'appuient sur les principes de base de la communication et de la résolution de problèmes associés à la satisfaction conjugale. Markman et ses collaborateurs ont sans doute produit l'ensemble de recherches le plus important dans le domaine de la prévention des conflits conjugaux, ayant même fait une relance de neuf ans. De fait, cette intervention est le seul programme préventif qui a été évalué au-delà d'une période de 18 mois. Parmi les résultats que Markman et son équipe ont obtenus, les plus probants indiquent que les sujets du groupe expérimental améliorent davantage leurs habiletés de communication et qu'ils sont plus satisfaits que les sujets du groupe contrôle (Floyd et collab., 1995). 
Une équipe de chercheurs de l'Université Laval, composée de Jean-Marie Boisvert ${ }^{5}$, Robert Ladouceur ${ }^{6}$ et Madeleine Beaudry ${ }^{7}$, évalue une adaptation québécoise de ce programme pour les jeunes couples. Nous avons utilisé cette dernière version et l'avons modifiée pour qu'elle puisse mieux répondre aux besoins des couples de familles recomposées. L'une des principales modifications a été d'ajouter deux sessions ${ }^{8}$ au programme original afin de pouvoir offrir de l'information pertinente sur le fonctionnement d'une famille recomposée. Plus précisément, les objectifs du programme d'intervention préventive adaptée aux couples de familles recomposées sont :

1. Apprendre aux conjoints à communiquer efficacement et à exprimer leurs sentiments

Une communication interpersonnelle efficace implique que chaque personne exprime clairement son message et que chacun s'assure à la fois d'avoir bien compris l'autre et d'avoir été bien compris par l'autre. De plus, les sentiments sont exprimés de façon précise et non menaçante. Le programme comporte des rencontres qui ont pour but de développer, d'une manière systématique, les habiletés de base d'une bonne communication. La capacité des conjoints à bien communiquer devrait diminuer les risques de conflits (Beaudry et Boisvert, 1988).

2. Sensibiliser les conjoints à leurs attentes respectives concernant la nouvelle famille et la relation de couple ainsi qu'aux conséquences qu'elles ont sur leur satisfaction

II semble important, pour les couples de familles recomposées, qu'ils définissent leurs attentes respectives, qu'ils les expriment et en discutent avant de chercher à faire des compromis. Cette procédure

5. M. Boisvert est professeur et chercheur à l'École de psychologie.

6. M. Ladouceur est professeur et chercheur à l'École de psychologie.

7. $M^{\text {me }}$ Beaudry est professeure et chercheure à l'École de service social.

8. Au départ, le programme PREP et son adaptation pour les jeunes couples québécois comportaient six sessions de deux heures et demie chacune. L'adaptation du programme pour les couples de familles recomposées en comprend huit de la même durée. 
pourrait les aider à évaluer le réalisme de leurs attentes, à diminuer la tension de rôle, de même que les émotions négatives et les conflits associés à des attentes irréalistes ou non exprimées. De plus, le fait de discuter des attentes quant au partage des responsabilités peut favoriser un partage plus équitable sur ce plan.

3. Favoriser l'acceptation des situations propres à une recomposition familiale

L'approche behaviorale cognitive que nous préconisons propose d'aider les conjoints à accepter ce qu'ils ne peuvent changer dans une famille recomposée (ex. : vivre dans une famille différente de celle dont les conjoints rêvaient). Des informations pertinentes concernant le fonctionnement familial, les difficultés habituellement rencontrées dans les premières phases d'une recomposition et les forces que renferme cette forme familiale serviront à rassurer les conjoints sur ce qu'ils vivent et ressentent. À travers les discussions qui portent sur ces sujets, les conjoints ont la possibilité de se construire une perspective familiale valorisante et adaptée à leur situation. Nous croyons qu'ils pourront ainsi mieux accepter les différences.

\section{Encourager le couple à faire des activités agréables ensemble}

Les couples en détresse font moins d'activités plaisantes ensemble que ceux qui fonctionnent bien. Dans la famille recomposée, les couples ont souvent de la difficulté à se réserver du temps pour passer des moments agréables ensemble, ce qui ne favorise pas l'intimité et la stabilité de la relation. Afin d'augmenter la satisfaction conjugale, le programme incitera les couples à enrichir les aspects positifs de leurs relations en les encourageant à faire des activités agréables pour eux entre les rencontres.

5. Apprendre aux couples des stratégies constructives de résolution de problèmes

La vie en famille recomposée comporte un potentiel élevé de conflits. Pour faire face à ces difficultés, une méthode efficace de résolution de conflits sera enseignée. Chaque couple la mettra immédiatement en pratique en choisissant un problème réel qu'il éprouve. 
6. Sensibiliser les couples aux difficultés que vivent les enfants et les aider à faire face à ces difficultés

Une des dimensions importantes de ce programme consiste à aider les conjoints à comprendre les difficultés particulières que vivent les enfants de familles recomposées. Pour faire face aux difficultés, les couples seront encouragés à mettre en pratique leurs habiletés de communication et de résolution de problèmes tant entre eux qu'avec leurs enfants. Nous souhaitons ainsi contribuer à améliorer les relations parents-enfants, et particulièrement les relations beauxparents / enfants.

\section{Encourager les conjoints à s'offrir un soutien mutuel}

Le bien-être de chaque conjoint est intimement lié au soutien qu'il peut donner et recevoir. Une vision du couple où la courtoisie, l'amitié et le partage sont des dimensions essentielles ne peut que favoriser le soutien. En effet, le développement d'un esprit d'équipe est un moyen positif d'accroître l'engagement et d'amener les conjoints à être sensibles aux besoins de l'autre, à prendre en considération ses points de vue et à chercher à l'appuyer d'une manière constructive sans pour cela abandonner son identité, ses opinions et ses valeurs. Les couples qui partagent cette perspective devraient être plus conscients de leurs forces et plus satisfaits de leur relation.

8. Inciter les conjoints à maintenir ou à développer un réseau social adéquat

Les premières années de vie en famille recomposée imposent souvent des changements importants dans le réseau social de chaque conjoint. Chacun risque de s'isoler socialement. Pourtant, comme l'indique le ministère de la Santé et des Services sociaux, " le renforcement du réseau social des personnes présente un potentiel inestimable pour la prévention des problèmes de santé et des problèmes sociaux... " (1992, p. 149). Par les échanges en groupe, le programme incitera donc les conjoints à développer leur réseau (entre eux ou à l'extérieur du groupe), à reconnaître les ressources existantes dans leurs familles, dans leur communauté et à discuter des façons de s'y prendre pour obtenir diverses formes de soutien. 


\section{La structure du programme}

Ce programme est offert en session intensive pendant deux fins de semaine, à raison de cinq heures par jour le samedi et le dimanche. Avant chacune des fins de semaine, les couples sont invités à lire un manuel qui explique en détail les principes de base de la communication et du processus de la résolution des problèmes. Certaines informations sur la famille recomposée sont intégrées à ces explications, d'autres forment des sections distinctes du manuel.

Pendant les fins de semaine, les rencontres se divisent en deux temps. D'abord les participants reçoivent, en groupe, de l'information concernant des thèmes reliés à la famille recomposée ou encore en lien avec les objectifs spécifiques déjà cités. Ils échangent à ce sujet de façon à préconiser l'entraide et la valorisation des stratégies déjà élaborées par les couples pour surmonter leurs difficultés. Dans un deuxième temps, chacun des couples se retire du groupe. Durant cette période, les conjoints discutent avec un intervenant ou une intervenante et expérimentent de façon personnalisée les exercices de communication et de résolution de problèmes suggérés.

Le but des activités de la première fin de semaine est d'enseigner les habiletés de base de la communication, soit les principes de l'émetteur et du récepteur ainsi que l'expression des sentiments. Par la suite, les couples sont invités à discuter de leurs attentes face à leur relation conjugale et familiale en utilisant les habiletés de communication enseignées précédemment. En parallèle, les sessions de groupe sont utilisées pour favoriser l'entraide et le soutien entre pairs. Finalement, à la fin des deux premiers jours l'intervenant met l'accent sur le rôle de l'amitié et du plaisir dans le maintien de la stabilité et de l'intimité dans la relation. II incite les couples à trouver des activités plaisantes qu'ils pourront faire ensemble entre les deux fins de semaine. Les activités de la deuxième fin de semaine visent à consolider les habiletés de communication apprises lors de la première fin de semaine et à mettre en pratique un modèle de résolution de problèmes selon une structure permettant de mieux négocier les différends. L'intervenant aborde également les réactions particulières des enfants face à la recomposition. Les couples sont alors encouragés à appliquer les principes de base de la communication et de la résolution de problèmes avec leurs enfants. Enfin, ils sont invités à préciser de manière concrète la façon dont ils peuvent 
s'apporter du soutien et ils explorent leur réseau social et les différentes manières de le développer.

Une des caractéristiques intéressantes de ce programme, c'est son approche à la fois individuelle et de groupe. Dans le cas des couples de familles recomposées nous croyons que la combinaison de ces deux approches est mieux adaptée à leur situation familiale. En effet, si les couples de familles recomposées vivent des difficultés différentes de celles des familles nucléaires, ces difficultés peuvent également être différentes selon la structure spécifique de leur famille. Par exemple, un seul ou les deux conjoints peuvent avoir des enfants de familles antérieures; les enfants peuvent visiter ou demeurer dans la famille; la recomposition peut avoir lieu lorsque les enfants sont jeunes ou lorsqu'ils sont adolescents, etc. La variété structurelle de ces familles apporte des difficultés particulières qui obligent l'intervenant à individualiser son action. Construire un programme d'intervention standardisée qui viserait à inclure toutes les composantes spécifiques de cette forme familiale est, à notre avis, utopique. Par contre, éliminer l'approche de groupe, c'est aussi se priver de l'apport que cette forme d'intervention peut fournir.

Ces familles sont invisibles dans notre société. Qui peut dire si un adulte est le beau-père ou le père de l'enfant qui l'accompagne? C'est pourquoi les individus qui les composent ressentent souvent de l'isolement et sont fréquemment perdus par rapport aux comportements qu'ils doivent adopter. Le soutien du groupe, le partage des difficultés communes et les échanges de stratégies ne sont pas à négliger pour des couples qui n'ont pas de guides sociaux pour leur permettre de savoir comment agir. À ce sujet, une recherche récente indique qu'un facteur important de la prédiction de la qualité de la relation entre une belle-mère et un adolescent est la capacité de la famille à trouver le soutien nécessaire à travers les relations, les amis ou les voisins (Quick, Newman et McKenry, 1995). En conséquence, un programme d'intervention préventive conçu pour les couples de familles recomposées doit idéalement inclure : 1. une dimension individuelle permettant d'adapter l'intervention aux difficultés particulières de chacun des couples et 2 . une dimension de groupe permettant de briser l'isolement tout en favorisant le soutien entre pairs et le partage d'informations. À notre avis, la méthode mixte est une combinaison gagnante dans ce contexte. 


\section{L'ÉVALUATION DU PROGRAMME}

L'élaboration d'un programme préventif adapté aux couples de familles recomposées est la première étape d'un projet plus vaste qui vise à en évaluer les effets auprès des conjoints. Cette deuxième étape s'effectuera dans le cadre de la thèse de doctorat de l'auteure principale. L'évaluation est prévue à l'hiver 1998 et elle fait suite à une préexpérimentation du programme qui est actuellement en cours. La section qui suit présente très brièvement la méthode d'évaluation qui sera utilisée.

L'hypothèse centrale de la recherche est que le programme contribuera à augmenter les habiletés de communication de chacun des conjoints. Afin de vérifier cette hypothèse nous comptons utiliser un devis de type expérimental à cas unique à niveaux de base multiples en fonction des individus. Cette forme d'évaluation comporte plusieurs avantages : 1) ce protocole est particulièrement indiqué pour vérifier l'efficacité d'une procédure clinique; 2 ) il est tout à fait approprié lorsque le recrutement d'un large échantillon de sujets se révèle difficile ${ }^{9}$ (Ladouceur et Bégin, 1980) et 3 ) il a l'avantage de ne pas nécessiter d'arrêt de traitement (Sheldon, 1983). De plus, l'utilisation d'un protocole à cas unique permet de mesurer l'efficacité de l'intervention pour chacun des couples et même pour chacun des conjoints. En effet, si les méthodes comparatives entre groupes peuvent donner des guides généraux concernant la sorte de traitement qui peut aider les clients, le protocole à cas unique permet d'obtenir des informations très précises sur quel type d'intervention aide quel type de client en particulier et pour quel type de problème très spécifique (Nelson, 1988; Witkin et Harrison, 1979). Enfin, ce modèle permet une évaluation très sensible de l'efficacité de l'intervention tout au long du processus. L'utilisation des mesures répétées (principale caractéristique de ce modèle) aide à identifier les variations inattendues ou inhabituelles au fur et à mesure que l'évaluation se poursuit, et à évaluer si elles sont reliées au traitement ou à des facteurs extérieurs (Witkin et Harrison, 1979).

9. La population de familles recomposées au Québec est estimée à $8,4 \%$ (Bellerose et collab., 1994). Bien qu'elle soit en progression, il ne nous semble pas réaliste de croire que nous pourrons facilement recruter un large échantillon dans la seule région de Québec. 
Le protocole à cas unique à niveaux de base multiples en fonction des individus est un protocole qui comporte un modèle de type $A B(A=$ niveaux de base, $B=$ intervention) appliqué à plusieurs clients. La première étape consiste à mesurer le comportement (communication) qui va servir à noter les taux de base. Dans ce cas-ci, les conjoints notent quotidiennement sur une échelle de 1 à 7 le déroulement des discussions qui ont lieu avec leur conjoint ou conjointe ${ }^{10}$ ainsi que l'impact que ces discussions ont sur eux. Ces données établissent la qualité de la communication entre les conjoints avant l'intervention. L'intervention est introduite dès que quatre couples obtiennent un niveau de base stable (le processus se répétera pour deux autres groupes de quatre couples). Nous prévoyons qu'environ trois semaines de mesures quotidiennes seront nécessaires pour établir un niveau de base stable. Ces mesures se poursuivront à un même rythme durant toute la durée de l'intervention. C'est la comparaison entre la représentation graphique des données obtenues avant et pendant l'introduction de l'intervention qui permettra de mesurer les changements dus à l'intervention pour les couples (Nelson, 1988, p. 377-396).

Trois autres instruments seront utilisés pour compléter l'évaluation des effets du programme : 1. Le test des habiletés de communication de Floyd et Markman (1984) sert à évaluer la communication du point de vue d'observateurs externes; 2 . L'inventaire des difficultés rencontrées par les couples en famille recomposée (Beaudry, Parent et Saint-Jacques, à paraître ${ }^{11}$ ) permet de cibler les

10. L'intervenant remplit avec chacun des conjoints séparément un guide de référence personnalisé qui lui sert ensuite à remplir ses fiches d'observations quotidiennes. Le score 1 correspond au déroulement de la pire discussion que la personne peut avoir avec son partenaire, le score 3 à ce qui se passe habituellement, le score 5 à ce qui se passe au mieux. Le score 7 correspond au déroulement de la discussion idéale; c'est le but à atteindre dans l'intervention. En conséquence, la personnalisation de l'instrument signifie que, pour certains, le score 1 correspondra à une dispute, alors que pour d'autres ce score sera associé à un silence absolu entre les conjoints.

11. Nous achevons présentement la validation de ce questionnaire auprès d'un échantillon de 100 hommes et 100 femmes vivant la recomposition de leur famille. Les résultats préliminaires indiquent un facteur unique pour chacune des quatre échelles. Les alphas sont : 0,84 à l'échelle 1 (dimension sociale et familiale); 0,80 à l'échelle 2 (situations reliées au rôle de conjoint); 0,90 à l'échelle 3 (situations reliées au rôle de parent); 0,89 à l'échelle 4 (situations reliées au rôle de beau-parent). 
difficultés des couples ainsi que leur désir de changement, et 3. Le Questionnaire d'adaptation à la vie conjugale de Locke et Wallace (1959), traduit par Wright et Sabourin (1985), mesure la satisfaction des conjoints par rapport à leur vie de couple.

L'évaluation de ce programme comporte également une dimension plus qualitative. Tout au long de l'intervention, les intervenants seront interrogés sur leurs perceptions en rapport avec: 1 ) le déroulement des rencontres et de chacune des activités; 2) la participation et la réaction des conjoints; 3) l'animation, la réaction et les problèmes rencontrés par les intervenants; 4) la pertinence du matériel et 5) les modifications éventuelles. En ce qui concerne les participants, ils répondront à deux questions ouvertes servant à vérifier ce qui a été le plus aidant et le moins aidant dans le programme et ils seront également interrogés sur leur degré d'implication dans le programme (ex. : Dans quelle mesure les tâches à la maison ontelles été effectuées?).

\section{CONCLUSION}

L'élaboration et l'évaluation d'un programme d'intervention préventive pour les couples de familles recomposées est une première au Québec. En fait, les recherches dans ce domaine sont encore jeunes et peu de praticiens ont une expertise dans ce champ spécifique. Pour cette raison, les quelques individus qui s'intéressent à ce domaine actuellement au Québec peuvent être qualifiés de précurseurs.

Par ailleurs, si la nouveauté est attirante, elle est également très exigeante pour ses adeptes. Les chercheurs doivent tenir compte dans leur devis de recherche d'une problématique de relations complexes dont les effets sur les membres de la famille sont encore mal connus. Ils doivent également adapter leurs instruments de mesure ou en créer de nouveaux. Quant aux praticiens, ils voient de plus en plus la nécessité d'ajuster leurs interventions aux demandes d'aide et aux besoins spécifiques de cette population. De plus, tous doivent sortir des cadres de référence habituels de la famille pour en arriver à comprendre l'ensemble des forces en interaction et en opposition dans une recomposition familiale.

Dans cette difficile et longue traversée qui mène à la connaissance, il est indéniable que les praticiens autant que les chercheurs 
peuvent aider à éclairer ce qui n'est encore que brouillard et ombre. Mieux encore, si les praticiens et les chercheurs font l'effort de se consulter au sujet de leurs travaux respectifs, peut-être que le développement des connaissances dans le domaine de la recomposition familiale pourra faire un bond important au Québec. C'est avec cet espoir que nous poursuivons la réalisation de ce projet d'intervention préventive pour les couples qui vivent la recomposition de leur famille. D'un côté, nous souhaitons pouvoir profiter de l'expertise des praticiens en tant que spécialistes de l'intervention, de l'autre nous espérons pouvoir leur apporter des outils de référence utiles et des guides pratiques à utiliser dans leurs interventions futures. Quant à l'évaluation du programme, elle contribuera à augmenter les connaissances sur la prévention des conflits conjugaux et sur l'efficacité de ce type d'intervention dans les périodes de transition. Ainsi, ces résultats permettront d'élargir le choix des actions possibles pour tous ceux qui interviennent auprès des membres de ces familles.

Les connaissances générées par l'ensemble de ce projet, de même que les alliances susceptibles d'être établies entre les praticiens et les chercheurs, engendrent l'espoir que de plus en plus de chercheurs en service social s'intéressent à l'intervention. Idéalement, nous souhaitons qu'au-delà des quelques échanges qui existent actuellement entre les praticiens et les chercheurs il puisse y avoir des lieux d'exercice permettant une association véritable et durable entre la recherche et la pratique. Dans une profession dont les racines sont si bien ancrées dans l'intervention, n'est-ce pas un vœu légitime? C'est du moins le rêve que nous caressons pour l'avenir.

Claudine PARENT

Étudiante au doctorat

École de service social Membre étudiante du Centre de recherche sur les services communautaires Université Laval

Madeleine BEAUDRY

Professeure

École de service social Chercheure au Centre de recherche sur les services communautaires 


\section{Références bibliographiques}

BEAUDRY, M. et J.-M. BOISVERT (1988). Psychologie du couple. Quand la science se met à parler d'amour, Montréal, Éditions du Méridien.

Bellerose, C., C. LAVAlléE et J. CAMIRAND (1994). Enquête sociale et de santé 1992-1993. Faits saillants, Gouvernement du Québec.

CHERLIN, A. (1987). « Dix ans après le remariage comme institution incomplète ", Dialogue 97, p. 65-68.

Cligempeel, G.W., R. IEVOLI et E. BRAND (1984). " Structural complexity and the quality of stepfather-stepchild relationships ", Family Process 23, p. 547-560.

CONSEIL DE LA FAMILLE (1995). La famille... composée autrement, Gouvernement du Québec.

Crosbie-BurnetT, M. (1989). "Application of family stress theory to remarriage : A model for assessing and helping stepfamilies ", Family Relations 38, p. 323-331.

Desrosiers, H., C. Le Bourdais et B. LAPLANTE (1995). " Les dissolutions d'union dans les familles recomposées : L'expérience des femmes canadiennes ", Recherches sociographiques, vol. XXXVI, n 1, p. 47-64.

DUNCAN, S.F. et G. Brown (1992). "Renew : A Program for Building Remarried Family Strengths ", Families in Society: The Journal of Contemporary Human Services, p. 149-158.

FAUSSE, D.F. (1995). "Stress Inoculation Training for Stepcouples ", Marriage and Family Review, vol. 21, nos 1/2, p. 137-155.

FLOYD, F.J. et H.J. MARKMAN (1984). " An economical observational measure of couple's communication skill ", Journal of Consulting and Clinical Psychology 52, p. 97-103.

Floyd, F.J., H.J. Markman, S. Kelly, S.L. Blumberg et S.M. Stanley (1995). "Preventive intervention and relationship enhancement ", dans Neil S. Jacobson et Alan S. Gurman (dir.), Clinical Handbook of Couple Therapy, New York, Guilford Press, p. 212-226.

FORTIER, F. et G. MARINO (1990). « Un modèle développemental pour l'intervention auprès des familles recomposées ", Service social, vol. 39, $n^{\circ} 3$, p. 112-134.

GaNong, H.L. et M. Coleman (1994). Remarried Family Relationships, Thousand Oaks, Sage Publications. 
HeTHeRington, M.E., M. Cox et R. Cox (1985). " Long-term effects of divorce and remarriage on the adjustment of children ", Journal of the American Academy of Child Psychiatry 24, p. 518-530.

HOBART, C.W. (1988). « Perception of parent-child relationship in first married and remarried families ", Family Relations 37, p. 175-182.

JACOBSON, D. (1990). "Stress and support in stepfamily formation : The cultural context of social support ", dans B.R. Sarason, I.G. Sarason et G. Pierce (dir.), Social Support : An Interactional View, New York, John Wiley \& Sons, p. 199-218.

LADOUCEUR, R. et G. BÉGIN (1980). Protocoles de recherche en sciences appliquées et fondamentales, Saint-Hyacinthe, Edisem.

LAWTON, J.M. et M.R. SANDERS (1994). " Designing effective behavioral family interventions for stepfamilies ", Clinical Psychology Review, vol. 14, nº 5, p. 463-496.

LESLIE, L.A. et N. EPSTEIN (1988). "Cognitive-Behavioral treatment of remarried families ", dans N. Epstein, S.E. Schlesinger et W. Dryden (dir.), Cognitive-Behavioral Therapy with Families, New York, Bruner/ Mazel Inc., p. 151-183.

MARKMAN, H.J. (1991). " Backwards into the future of couples therapy and couples therapy research ", Journal of Family Psychology, vol. 4, $n^{\circ} 4$, p. 416-425.

MARKMAN, H.J. (1981). "Prediction of marital distress : A 5-year followup ", Journal of Consulting and Clinical Psychology 49, p. 760-762.

MINISTÈRE DE LA SANTÉ ET DES SERVICES SOCIAUX (1992). Loi sur les services de santé et les services sociaux, Québec, Gouvernement du Québec.

NELSON, J.C. (1988). " Single-subject research », dans R.M. Grinnell Jr. (dir.), Social Work Research and Evaluation ( $3^{\ominus}$ éd.), Faculty of Social Welfare, University of Calgary, Peacock Publishers, p. 362-400.

O'FLAHERTY, K.M. et L. WORKMAN EELLS (1993). "The development of remarriage-preparation programs ", Journal of Divorce and Remarriage, vol. 20, nos 1/2, p. 229-245.

Palisi, B.J., M. ORLEANS, D. CADdell et B. Korn (1991). « Adjustment to stepfatherhood: The effects of marital history and relations with children ", Journal of Divorce and Remarriage 14, p. 89-106.

PARENT, C. (1994). Étude exploratoire des stratégies d'adaptation utilisées par les beaux-parents en famille recomposée, Mémoire présenté pour l'obtention du grade de maître en service social, Faculté des études supérieures, Université Laval, juin. 
PASley, K., M.G. KoCH et M. IHINGer-Tallman (1993). " Problems in remarriage : An exploratory study of intact and terminated remarriages ", Journal of Divorce and Remarriage, vol. 20, $n^{\text {os }} 1 / 2$, p. 63-83.

QUICK, D.S., B.M. NEWMAN et P.C. MCKENRY (1995). "Influences on the quality of the stepmother-adolescent relationship ", Journal of Divorce and Remarriage, vol. 24, nos 1/2, p. 99-114.

SAINT-JACQUES, M.-C. (1993). La tension de rôle chez les familles recomposées : Étude des facteurs associés, Mémoire de maîtrise publié, Série " Résultats de recherche ", Laboratoire de service social, Université Laval.

SeCrétariat À la famille (1995). Les familles au Québec. Principales statistiques, Gouvernement du Québec, Ministère du Conseil exécutif, mars.

SHELDON, B. (1983). "The use of single case experimental designs in the evaluation of social work ", British Joumal of Social Work 13, p. 477-500.

SKOPIN, A.R., B.M. NEWMAN et P. MCKENRY (1993). «Influences on the quality of stepfather-adolescent relationships : Views of both family members ", Journal of Divorce and Remarriage, vol. 19, nos 3/4, p. 181-196.

StATISTIQUe CANAdA (1994). Tendances sociales canadiennes, numéro $11-008 \mathrm{~F}$ au Catalogue.

VISHER, E.B. et J.S. VISHER (1990). « Dynamics of successful stepfamilies », Journal of Divorce and Remarriage, vol. 14, n० 1, p. 3-11.

WHITE, L.K. et A. BOOTH (1985). « The quality and stability of remarriage : The role of stepchildren ", American Sociological Review 50, p. 689-698. 